\title{
\begin{tabular}{l|l} 
Mitraries & DSpace@MIT
\end{tabular}
}

\author{
MIT Open Access Articles
}

\section{Higher-order photon correlations in pulsed photonic crystal nanolasers}

The MIT Faculty has made this article openly available. Please share how this access benefits you. Your story matters.

Citation: Elvira, D. et al. “Higher-order Photon Correlations in Pulsed Photonic Crystal Nanolasers." Physical Review A 84.6 (2011): [4 pages]. (C2011 American Physical Society.

As Published: http://dx.doi.org/10.1103/PhysRevA.84.061802

Publisher: American Physical Society

Persistent URL: http://hdl.handle.net/1721.1/70488

Version: Final published version: final published article, as it appeared in a journal, conference proceedings, or other formally published context

Terms of Use: Article is made available in accordance with the publisher's policy and may be subject to US copyright law. Please refer to the publisher's site for terms of use. 


\title{
Higher-order photon correlations in pulsed photonic crystal nanolasers
}

\author{
D. Elvira, ${ }^{1}$ X. Hachair, ${ }^{1}$ V. B. Verma, ${ }^{2}$ R. Braive, ${ }^{1}$ G. Beaudoin, ${ }^{1}$ I. Robert-Philip, ${ }^{1}$ I. Sagnes, ${ }^{1}$ B. Baek,${ }^{2}$ S. W. Nam, ${ }^{2}$ \\ E. A. Dauler, ${ }^{3}$ I. Abram, ${ }^{1}$ M. J. Stevens, ${ }^{2}$ and A. Beveratos ${ }^{1, *}$ \\ ${ }^{1}$ Laboratoire de Photonique et Nanostructures LPN-CNRS UPR20, Route de Nozay, F-91460 Marcoussis, France \\ ${ }^{2}$ National Institute of Standards and Technology, 325 Broadway, Boulder, Colorado 80305, USA \\ ${ }^{3}$ Lincoln Laboratory, Massachusetts Institute of Technology, Lexington, Massachusetts 02420, USA
}

(Received 10 June 2011; published 16 December 2011)

\begin{abstract}
We report on the higher-order photon correlations of a high- $\beta$ nanolaser under pulsed excitation at room temperature. Using a multiplexed four-element superconducting single-photon detector we measured $g^{(n)}(\overrightarrow{0})$ with $n=2,3,4$. All orders of correlation display partially chaotic statistics, even at four times the threshold excitation power. We show that this departure from coherence and Poisson statistics is due to the quantum fluctuations associated with the small number of photons at the lasing threshold.
\end{abstract}

DOI: 10.1103/PhysRevA.84.061802

PACS number(s): 42.55.Tv, 42.50.Ar, 42.50.Lc, 42.55.Ah

The physics of nanoscale lasers is expected to be radically different from that of conventional lasers, due to their very high spontaneous emission ratio $(\beta)$ into the lasing mode, approaching $\beta \rightarrow 1$. Because of the high $\beta$, the system is capable of lasing with a small number of photons in the cavity mode, of the order of $\beta^{-1 / 2}$ [1], and thus the stimulated emission threshold is low and the lasing turn-on time is expected to be very short. However, the quantum fluctuations associated with the small number of photons at threshold cause the output intensity of the laser to fluctuate strongly with chaotic statistics which can be present well above the stimulated emission threshold [2,3] and may even suppress continuous-wave lasing [4]. These small-number fluctuations do not necessarily obey Gaussian statistics (as is the case for large conventional lasers), and thus a measurement only of $g^{(2)}$ is not sufficient to characterize them. A study of these fluctuations requires measurement of the higher-order correlation functions of the laser output, as $g^{(2)}, g^{(3)}$, and $g^{(4)}$ give information on the variance, skewness, and kurtosis (respectively) of the fluctuation statistics. Understanding these quantum fluctuations is crucial for the operation of nanolasers, particularly if nanoscale lasers are to be used in demanding applications, such as interchip optical interconnects or integrated clocks.

Several studies on the dynamics and the noise characteristics of nanolasers have been reported in recent years. Measurements of the stationary second-order correlation function $g^{(2)}\left(\tau=t_{1}-t_{2}\right)$ were reported some years ago [5,6] and more recently the evolution of $g^{(2)}\left(t_{1}, t_{2}\right)$ was followed with picosecond resolution in semiconductor micropillar nanolasers operated at $4 \mathrm{~K}$ in the weak-coupling [7,8] or the strongcoupling [9] regimes. Lately, the noise properties of nanolasers operating at room temperature were also reported [10]. In most of these studies, the cavity volume was of the order of a cubic wavelength and the gain material consisted of a small number of semiconductor quantum dots. In some recent work on plasmonic structures, lasers with even smaller volumes have been reported [11-13], reducing further the number of emitting dipoles and photons in the cavity. Clearly, in such small lasers, and in view of the very small number

*alexios.beveratos@1pn.cnrs.fr of photons at threshold, the traditional laser theories based on the "thermodynamic limit" of large-ensemble averaging and continuum approximations for the gain medium and the electromagnetic field are no longer valid, as the discrete nature of the number of dipoles and photons must be taken into account explicitly.

In this Rapid Communication we report an experimental study of the higher-order photon autocorrelation functions of a single-mode photonic crystal nanocavity laser, with quantum dots as its gain material, operating at room temperature, in the telecommunications wavelength range, and under pulsed excitation. In the second, third, and fourth orders of correlation, the output intensity fluctuations display a chaotic component and deviate from standard Poisson statistics, even at pump powers of over four times the threshold. These noise properties can be accounted for by the quantum fluctuations arising from the small number of photons at threshold, through a simple model that provides insight into the physics of nanolasers.

The nanolaser cavity is formed by a photonic crystal double heterostructure [14] etched on a 320-nm-thick suspended InP membrane [10], grown by metalo-organic chemical vapor deposition and incorporating a single layer of self-assembled InAs $_{1-x} \mathrm{P}_{x}$ quantum dots [15] at its vertical center plane (See Fig. 1). The quantum dot density is of the order of $1.5 \times$ $10^{10} \mathrm{~cm}^{-2}$ and their spontaneous emission is centered at $\sim 1560 \mathrm{~nm}$ at $300 \mathrm{~K}$ with an inhomogeneous linewidth of $\sim 150 \mathrm{~nm}$. The cavity is fabricated using electron beam lithography, inductively coupled plasma etching, and wet etching [16]. The structure consists of a W1 photonic crystal waveguide composed of one missing row of holes in the $\Gamma-K$ direction of a hexagonal lattice structure with a lattice constant of $a_{m}=410 \mathrm{~nm}$, and an air-hole radius of $r=0.293 a_{m}$. The lattice constant is modified over two periods at the center of the photonic crystal waveguide along the $\Gamma-K$ direction alone, to the value of $a_{l}=440 \mathrm{~nm}$, thus forming a nanocavity with an effective volume of $1.3(\lambda / n)^{3}$, enclosed by two surrounding "mirror" waveguides with a smaller lattice constant, and containing on average some 70 quantum dots. The quality factor of the cavity is obtained by measuring the coherence time of the device output when bleaching the quantum dot absorption [17] and by measuring the cavity ring-down time through the second-order autocorrelation function well above 


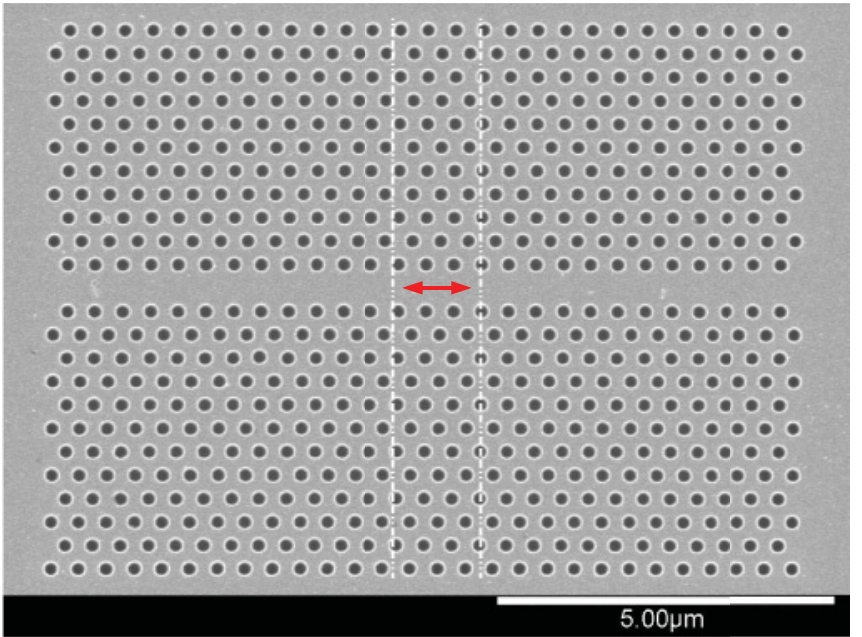

FIG. 1. (Color online) Double-heterostructure photonic crystal cavity. White dashed lines indicate the change in periodicity corresponding to the mirror position. The red arrow shows the laser cavity.

threshold. Both methods give a quality factor of $Q=45000$, corresponding to a cavity lifetime of $1 / \Gamma_{c}=35 \mathrm{ps}$.

The nanolaser is studied at room temperature. Optical excitation is provided by a pulsed Ti:sapphire laser emitting at $805 \mathrm{~nm}$ with a repetition rate of $82 \mathrm{MHz}$. The pump pulse is broadened to $\sim 50$ ps after passage through a $25-\mathrm{m}$ length of single-mode optical fiber, and is then focused to a $5-\mu \mathrm{m}$ spot on the sample with a microscope objective [numerical aperture (N.A.) $=0.4$ ]. When the quantum dots are highly excited and contain several electron-hole pairs, they emit into a broad spectrum with a lifetime of $1 / \gamma_{\|}=400 \mathrm{ps}$ $[10,18]$ which feeds the cavity mode [19]. Emission from the nanocavity laser is collected with the same objective, directed through a dichroic beam splitter that blocks the pump light, and is sent to a $0.75-\mathrm{m}$ grating monochromator. One output port of the monochromator holds an image-intensified near-infrared camera, which records the emitted spectrum and power. Alternatively, an internal mirror can be flipped, directing light to the other output port, where it is collected into a single-mode optical fiber and sent to the detector. The detector is a four-element superconducting nanowire single-photon detector (SNSPD) [20] in which four independent, singlephoton-sensitive elements are interleaved over a single spatial mode of the optical beam [21]. Each element has a system detection efficiency of $\sim 1 \%$ at $1550 \mathrm{~nm}$ and a dark count rate of $\sim 100 \mathrm{~Hz}$. The active area of the four-element SNSPD is matched to the mode diameter of the fiber, allowing all four interleaved nanowire elements to equally sample a single spatial mode [21,22]. Fast four-channel electronics record photon arrival times on each element. These time-tag data are post-processed to obtain multi-start, multi-stop correlation histograms between two, three, and four SNSPD elements. Details on the data processing, including the elimination of crosstalk among the interleaved detectors, are given in the Supplemental Material [23].

Figure 2 presents a typical set of normalized experimental three-photon coincidence data, extracted from the time-tagged arrivals of photons on the four multiplexed SNSPD detectors.

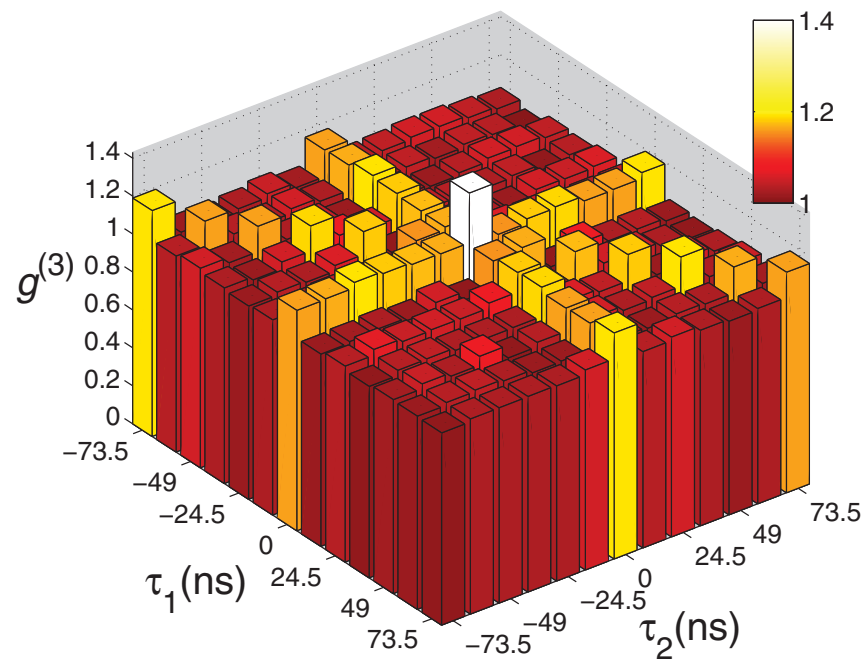

FIG. 2. (Color online) Third-order autocorrelation function $g^{(3)}\left(\tau_{1}, \tau_{2}\right)$ at a pump power $P=2 P_{\text {th }}$. The tallest bar, located at $\tau_{1}=\tau_{2}=0$, represents $g^{(3)}(0,0)$. The three ridges of elevated bars that fall along $\tau_{1}=0, \tau_{2}=0$, and $\tau_{1}=\tau_{2}$ correspond to $g^{(2)}(\overrightarrow{0})$. The remainder of the bars have an average value of $\sim 1$.

It corresponds to the third-order autocorrelation function $g^{(3)}\left(\tau_{1}, \tau_{2}\right)$ in histogram form.

For fully coherent light $g^{(n)}(\overrightarrow{0})=1$, whereas for chaotic (thermal) light $g^{(n)}(\overrightarrow{0})=n$ !, for all orders $n$. For partially coherent light, we may thus define

$$
h^{(n)}=\frac{g^{(n)}(\overrightarrow{0})-1}{n !-1},
$$

which is a normalized ratio, indicating whether the statistics are near coherent or chaotic: Below threshold the output light is chaotic, so that $h^{(n)}=1$, whereas above threshold, one expects a coherent laser output and $h^{(n)} \rightarrow 0$ for all $n$.

In Fig. 3 we present the experimental data and the theoretical curves (described below) for the light-in-light-out curve (right-hand axis) and $h^{(n)}$ for $n=\{2,3,4\}$ (left-hand axis) as a function of the pump power of the nanoscale laser under pulsed excitation. The traditional lasing threshold is clearly identified at the "knee" of the light-in-light-out curve $[1,3]$ (which corresponds to the inflection point of the $\log -\log$ curve), at $P_{\mathrm{th}}=100 \mu \mathrm{W}\left(=125 \mu \mathrm{J} \mathrm{cm}^{-2}\right.$ per incident pulse), while the $\beta$ factor is given by the ratio of the two slopes of the light-in-light-out curve (corresponding to the "jump" of that curve in log-log scale) of $\beta=0.008$. The $h^{(n)}$, which below threshold are equal to 1 , steadily decrease to reach $h^{(2)}=0.05 \pm 0.04, h^{(3)}=0.036 \pm 0.02$, and $h^{(4)}=$ $0.029 \pm 0.015$ at $P=4 P_{\mathrm{th}}=400 \mu \mathrm{W}$, all significantly higher than the value of 0 expected for coherent emission. The corresponding $g^{(n)}(\overrightarrow{0})$ are $g^{(2)}(\overrightarrow{0})=1.05 \pm 0.04, g^{(3)}(\overrightarrow{0})=$ $1.18 \pm 0.10$, and $g^{(4)}(\overrightarrow{0})=1.67 \pm 0.34$. In other words, the intensity fluctuations still have a chaotic component at four times the traditional threshold power, in sharp contrast to the behavior of conventional lasers (with $\beta \approx 10^{-5}$ ), for which all $h^{(n)}$ rapidly vanish above threshold.

The persistence of the chaotic component can be simply understood following Refs. [2-4]: Because of the small number of photons in the cavity at threshold (given by $\beta^{-1 / 2} \approx 11$ ), 


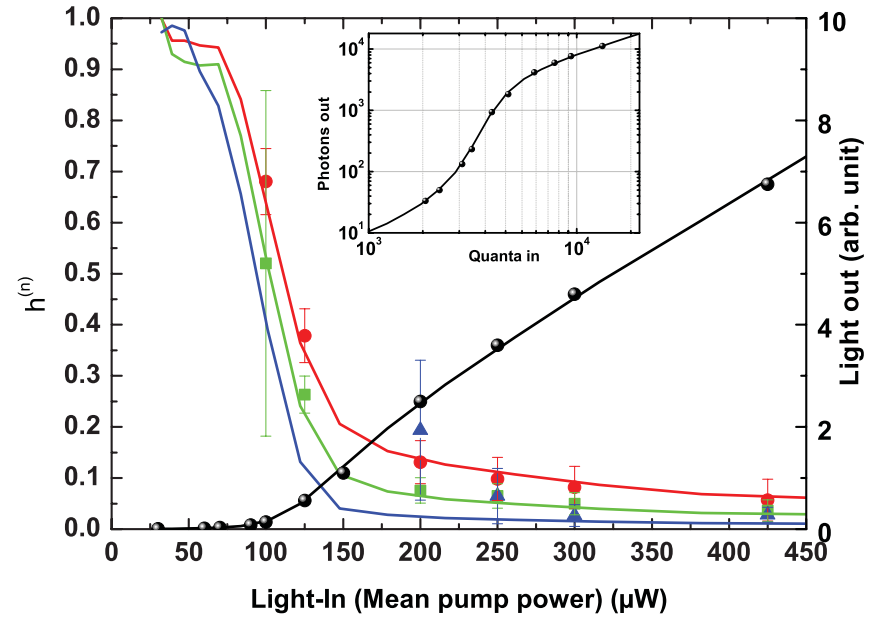

FIG. 3. (Color online) Right-hand axis: Experimental (black dots) and calculated (black line) light-in-light-out curve. Left-hand axis: Experimental (red circles, green squares, and blue triangles for $n=2,3,4$, respectively) and calculated (continuous lines of the corresponding colors) values of $h^{(n)}$ as functions of pump power. The fit of the $h^{(n)}$ data has no free parameters. Inset: Theoretical light-in-light-out curve in log-log scale. The experimental points have been normalized by the fit parameters.

a fluctuation of a few units introduces a non-negligible change in the stimulated emission rate and in the triggering of lasing, inducing chaotic (thermal) statistics even above threshold.

More quantitatively, we may model our system in terms of the traditional laser rate equations [3] under the constraint that the number of excited dipoles $N$ and the number of photons in the cavity $s$ be integers:

$$
\begin{gathered}
\frac{d N(t)}{d t}=P f(t)-\gamma_{\|} N(t)-\beta \gamma_{\|} N(t) s(t), \\
\frac{d s(t)}{d t}=-\Gamma_{c} s(t)+\beta \gamma_{\|} N(t)+\beta \gamma_{\|} N(t) s(t),
\end{gathered}
$$

where $f(t)$ is the temporal profile of the pump pulse intensity, of integral equal to one. For each incident power $P$, the rate equations are solved numerically by iterating over a time step $d t$, which is small compared with the characteristic times of the system $1 / \gamma_{\|}$and $1 / \Gamma_{c}$, and using a quantum jump approach [24] to ensure that $N$ and $s$ are integers: At each iteration step, the right-hand sides of Eqs. (2) and (3) are evaluated, taking into account the discrete evolution of $N$ and $s$ through a sequence of three binomial processes. First, the number of dipoles having decayed between $t$ and $t+d t$ is drawn from a binomial distribution with probability $p_{1}=\gamma_{\|}[1+\beta s(t)] d t$ for each one of them to decay. Second, the decaying dipoles each produce a photon that has a probability $p_{2}=\frac{[1+s(t)] \beta}{1+\beta s(t)}$ of entering the cavity. And third, the photons that have accumulated in the cavity can escape with probability $p_{3}=\Gamma_{c} d t$. For consistency, we also take into account Poissonian statistics for the pump, although it does not alter the overall conclusions. This algorithm is a variation of those developed for modeling population dynamics in biology (where populations can only consist of integer numbers of individuals) [25], however, with binomial rather than Poisson statistics, because each of the three processes is a partition.
For each incident power $P$, Eqs. (2) and (3) are solved many times (typically 1500), each solution being referred to as a "realization" of the laser operation. The $g^{(n)}(\overrightarrow{0})$ are calculated as

$$
g^{(n)}(\overrightarrow{0})=\frac{\left\langle\int_{t} \prod_{k=0}^{k=n-1}[s(t)-k]\right\rangle}{\left(\left\langle\int_{t} s(t)\right\rangle\right)^{n}},
$$

where $\langle x\rangle$ denotes the average of $x$ over all 1500 realizations (individual solutions). As can be seen in Fig. 3, the experimental data are very well described by our model without any adjustable parameters for the light-in-light-out curve as well the second- and third-order correlation functions. They are reasonably well described for the fourth-order correlation function, considering the large error bars of the measurements of $g^{(4)}(\overrightarrow{0})$.

For traditional lasers, quantum noise is introduced in the classical rate equations by means of a Langevin driving force with Gaussian statistics $[2,3]$. This approach, however, is not suited for high- $\beta$ lasers, for which threshold is attained with only a few photons in the cavity. The reason is that the fluctuation statistics are Gaussian only in the limit of large numbers of photons (the well-known central limit theorem). Also, since the populations can take only non-negative integer values, Langevin forces cannot adequately describe the fluctuations of small numbers of photons and dipoles as they do not take into account the discrete nature of the particles and permit excursions into negative numbers. In addition, Gaussian statistics imply that the higher-order correlation functions are sums of products of lower-order averages [26], a condition which is not verified for the actual small-laser statistics. Alternatively, for small lasers, the discretization of the numbers of dipoles and photons is usually treated through a set of master equations for the probabilities of states with a given number of excited dipoles $N$ and photons $s$ [4]. This approach can be readily implemented in the steady-state regime [6]. However, for pulsed lasers, it can only be used for very small systems: It rapidly becomes cumbersome as the system increases in size, since it requires calculating the time evolution of a matrix of $N \times N$ interdependent probabilities. On the other hand, our method requires the computation of independent trajectories (realizations) with at most $N$ jumps. Beyond the calculational simplicity this allows for a more intuitive approach to understanding the underlying physical phenomena.

To gain better insight into the effects of fluctuations, we present in Fig. 4 the evolution of the number of photons $s(t)$ for four realizations of the rate equations (dashed lines) at an excitation power $P=1.8 P_{\text {th }}$ as well as the mean of $s(t)$ over 1500 realizations (solid line). We observe that the individual realizations are relatively smooth curves with little noise, but display strong intensity and timing (jitter) fluctuations, which are correlated: The longer it takes for the pulse to be formed, the smaller is its intensity, because in the meantime the number of excited dipoles has decreased. This jitter implies that the threshold is crossed at random times, confirming that it is the fluctuations associated with the small number of photons at threshold that produce the excess noise. The inset of Fig. 4 represents the statistical distribution of the integrated pulse area (in number of photons), compared with 


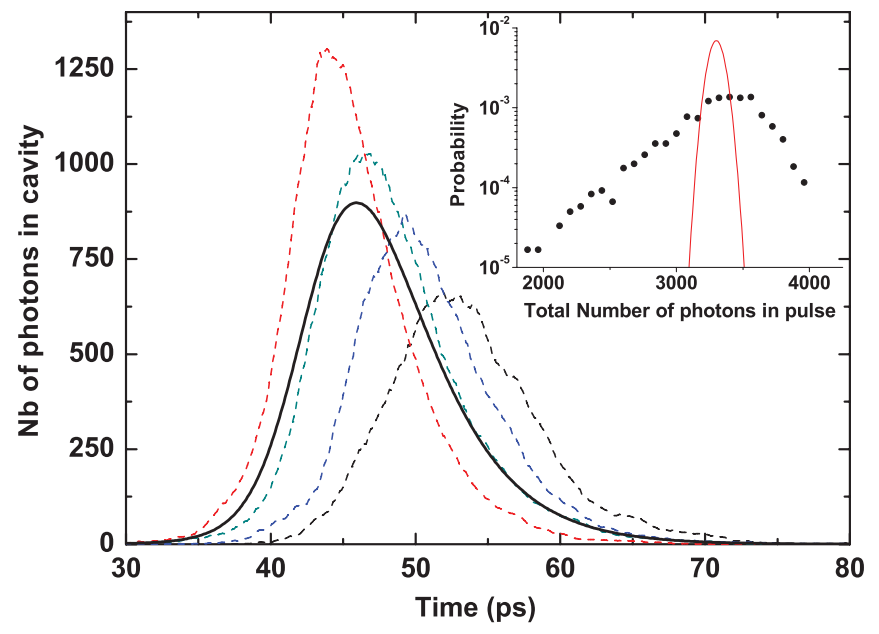

FIG. 4. (Color online) Dashed lines: Four different realizations of the stochastic rate equations at $P=1.8 P_{\text {th }}$. Solid line: The mean pulse averaged over 1500 realizations. It can be clearly seen that the longer it takes for the pulse to emerge, the smaller is its amplitude. Inset: The dots show the statistical distribution of the total number of photons per pulse at $P=1.8 P_{\mathrm{th}}$, and the line shows the expected distribution for Poisson statistics of the same mean.

the Poisson distribution of the same mean. As can be seen in the inset, the distribution of the number of photons in a pulse is broader than a Poisson distribution and displays a lower probability for high-intensity events and a higher probability for low-intensity events. It cannot be fitted as the superposition of a Poisson and a thermal distribution, thus underscoring the non-Gaussian character of the small-number photon fluctuations. The correlation between the intensity fluctuations and the jitter implies that late starts are more probable than early starts, even though the gain medium can provide more photons at earlier times. The physical origin of this effect may be similar to the suppression of continuous-wave lasing due to fluctuations in small lasers, discussed by Roy-Choudhoury et al. [4]. Fluctuations may suppress a nascent laser pulse, leading to "false starts" and delaying the time when the successful laser pulse takes off. The increased width of the statistics, the asymmetry (skewness), and the large tails (kurtosis) impact, respectively, the second-, third-, and fourth-order autocorrelation functions, and cause all $g^{(n)}(\overrightarrow{0})$ to deviate from the value of $g(n)(0)=1$, which is characteristic of coherent emission.

In conclusion, we measured up to the fourth-order autocorrelation function for a photonic crystal nanolaser operating at room temperature under pulsed excitation. We observe that all values of the autocorrelation function are significantly above unity, even at four times threshold, indicating the presence of chaotic fluctuations in spite of the predominance of stimulated emission. A model that takes into account the discrete nature of the number of photons and dipoles successfully describes the experimental data, providing an understanding of the operation of the nanolasers. Measurement of the higher-order photon correlation functions thus opens the way to the study of very small lasers, whose operation deviates strongly from the "thermodynamic limit" that governs conventional lasers.

The authors acknowledge financial support from the Triangle de la Physique under the BIRD project and from the French National Research Agency (ANR) through the Nanoscience and Nanotechnology Program (Project NATIF ANR-09-NANO-P103-36).
[1] P. R. Rice and H. J. Carmichael, Phys. Rev. A 50, 4318 (1994).

[2] H. F. Hofmann and O. Hess, J. Opt. Soc. Am. B 17, 1926 (2000).

[3] N. J. van Druten et al., Phys. Rev. A 62, 053808 (2000).

[4] K. Roy-Choudhury, S. Haas, and A. F. J. Levi, Phys. Rev. Lett. 102, 053902 (2009).

[5] S. M. Ulrich et al., Phys. Rev. Lett. 98, 043906 (2007).

[6] Y.-S. Choi et al., Appl. Phys. Lett. 91, 031108 (2007).

[7] M. Assmann et al., Phys. Rev. B 81, 165314 (2010).

[8] M. Assmann et al., Science 325, 297 (2009).

[9] J. Wiersig et al., Nature (London) 460, 245 (2009).

[10] R. Hostein et al., Opt. Lett. 35, 1154 (2010).

[11] M. A. Noginov et al., Nature (London) 460, 1110 (2009).

[12] M. T. Hill et al., Opt. Express 17, 11107 (2009).

[13] M. P. Nezhad et al., Nat. Photonics 4, 395 (2010).

[14] B.-S. Song et al., Nat. Mater. 4, 207 (2005).
[15] A. Michon et al., J. Appl. Phys. 104, 043504 (2008).

[16] A. Talneau et al., Appl. Phys. Lett. 92, 061105 (2008).

[17] R. Hostein et al., Appl. Phys. Lett. 94, 123101 (2009).

[18] D. Elvira et al., Phys. Rev. B 84, 195302 (2011)

[19] M. Winger et al., Phys. Rev. Lett. 103, 207403 (2009).

[20] G. N. Goltsman et al., Appl. Phys. Lett. 79, 705 (2001).

[21] E. A. Dauler et al., J. Mod. Opt. 56, 364 (2009).

[22] M. J. Stevens et al., Opt. Express 18, 1430 (2010).

[23] See Supplemental Material at http://link.aps.org/supplemental/ 10.1103/PhysRevA.84.061802 for a detailed analysis.

[24] M. B. Plenio and P. L. Knight, Rev. Mod. Phys. 70, 101 (1998).

[25] H. G. Solari and M. A. Natiello, Phys. Rev. E 67, 031918 (2003).

[26] P. A. Lemieux and D. J. Durian, J. Opt. Soc. Am. A 16, 1651 (1999). 